Proyecciones

Vol. $13 N^{\circ} 1$, pp.09-17 July 1994

Universidad Católica del Norte

Antofagasta - Chile

\title{
GLOBAL STABILITY RESULTS ON AN EPIDEMIOLOGICAL MODEL WITH A CORE GROUP (A NOTE ON THE PAPER "LOCAL STABILITY RESULTS ON A MODEL FOR TYPHOID FEVER WITH A CORE GROUP") *
}

\author{
JORGE GONZÁLEZ-GUZMÁN
}

Universidad Católica de Valparaíso, Valparaíso - Chile.

\begin{abstract}
A SIRS epidemiological model with two subpopulations and vital dynamics is analyzed. Both subpopulations sizes are considered constant by assuming that the birth and the death rates are equal. We consider the case where one subpopulation is a core, that is a very infectious small group, responsible for a big fraction of the incidence. For this case thresholds are determined and the main equilibrium points for the four dimensional system are shown to be globally stable by using a known Theorem of Markus on asymptotically autonomous systems. This system models the dynamics of typhoid fever, where the core is the group of food handlers. The results presented in this work are an extension of those presented in [3]
\end{abstract}

- Research supported in part by DGIP under Project 124.747/94. 


\section{Introduction}

The spread of an infectious disease in a population depends not only on the character of the disease,but also on the structure and behavior of the population. Therefore the study of heterogencous epidemic models is very important. Many previous results have been obtained in this direction (See [3] for a brief bibliographical discussion of [7],[1], [14], [8], [9]). We can see that global stability of the endemic equilibrium for many of the analyzed models is still an open problem .

In the present paper we extend the results presented in [3] in two directions : first we can weaken the assumptions made in [3] in order to obtain only local stability, and second we can obtain global stability for both the disease free and the endemic equilibrium. We recall that the model is an epidemiological SIRS core-noncore model [5], where the infectious contacts of the core people with the noncore ones are responsible for most of the total incidence. Our aim is to model the spread of typhoid fever in a modern big city, where many poeple eat in restaurants. In this case the food handlers constitute the core group

\section{The Model}

Let $\mathrm{N}$ be the total size of the population, $N_{1}$ the size of the core and $N_{2}$ the size of the noncore. Then $N=N_{1}+N_{2} ; N_{i}=S_{i}+I_{i}+R_{i}, i=1,2$, where S, I, R denote the number of susceptibles, infectious and removed (immune non infectious )individuals of each subpopulation. We assume that both subpopulations $N_{1}$ and $N_{2}$ are constant by setting the birth rate equal to the death rate .Note that in the case of the core group, the birth and the death rate have the meaning of a rate of change of the food handlers, not necessarily equal to the birth and death rate of the population. Let $x_{i}=I_{i} / N_{i}, y_{i}=R_{i} / N_{i}, i=1,2 ;\left(\lambda_{i j}\right)$ is the transmission matrix, that is, $\lambda_{i j}$ is the average number of infectious contacts (also called adequate contacts,see [5] ) of an infective in group $j$ with persons in group $i$ per unit time. Let $p=N_{1} / N_{2}, \delta_{i}$ the birth (or death) rate of subpopulation $i, \gamma$ is the recovery removal rate $(1 / \gamma$ is the average infectious period ),$w_{i}$ is the rate of loss of temporary immunity for group $i$ and $v_{i}$ is the immunization rate by vaccination in group $i$. We can accept different values of duration of immunity because the vaccination on both groups can be different ( for example, food handlers can get more dose of vaccine).

The model is then given by the following four dimensional system of autonomous differential equations :

$$
\begin{aligned}
x_{1}^{\prime} & =\left(1-x_{1}-y_{1}\right)\left(\lambda_{11} x_{1}+\frac{\lambda_{12}}{p} x_{2}\right)-x_{1}\left(\delta_{1}+\gamma\right) \\
y_{1}^{\prime} & =v_{1}+\left(\gamma-v_{1}\right) x_{1}-\left(v_{1}+\delta_{1}+w_{1}\right) y_{1} \\
x_{2}^{\prime} & =\left(1-x_{2}-y_{2}\right)\left(\lambda_{22} x_{2}+\lambda_{21} p x_{1}\right)-x_{2}\left(\delta_{2}+\gamma\right) \\
y_{2}^{\prime} & =v_{2}+\left(\gamma-v_{2}\right) x_{2}-\left(v_{2}+\delta_{2}+w_{2}\right) y_{2}
\end{aligned}
$$

with the conditions: $x_{i}, y_{i} \geq 0 ; x_{i}+y_{i} \leq 1, i=1,2$. 
Let $D$ be the domain $x_{i}, y_{i} \geq 0 ; x_{i}+y_{i} \leq 1, i=1,2$ in the four dimensional space $R^{4}$. It is easy to see, by applying Nagumos's Lemma, that domain D is positively invariant for system (1). This system satisfies the existence, uniquenes and prolongability conditions so that solutions of (1) starting in $D$ exists for all time and remain in $D$. Thus the model is well-possed.

The qualitative analysis of system (1) is very difficult because of the dimension,even the equilibrium points are difficult to be found explicity. But if we assume that $\lambda_{12}=0$, then system (1) decomposes into two two-dimensional systems and a complete analysis can be made. We recall that in [3] the assumption was $\lambda_{12}=\lambda_{22}=0$. In fact, with the former supposition, the first two equations :

$$
\begin{aligned}
& x_{1}^{\prime}=\left(1-x_{1}-y_{1}\right) \lambda_{11} x_{1}-x_{1}\left(\delta_{1}+\gamma\right) \\
& y_{1}^{\prime}=v_{1}+\left(\gamma-v_{1}\right) x_{1}-\left(v_{1}+\delta_{1}+w_{1}\right) y_{1}
\end{aligned}
$$

can be solved independent on the other two variables. Once a suitable solution $x_{1}(t)$ is obtained, we can introduce it in the second part of the system :

$$
\begin{aligned}
& x_{2}^{\prime}=\left(1-x_{2}-y_{2}\right)\left(\lambda_{22} x_{2}+\lambda_{21} p x_{1}(t)\right)-x_{2}\left(\delta_{2}+\gamma\right) \\
& y_{2}^{\prime}=v_{2}+\left(\gamma-v_{2}\right) x_{2}-\left(v_{2}+\delta_{2}+w_{2}\right) y_{2}
\end{aligned}
$$

Note that this last system is not autonomous. System (2) correspond to the one group model analyzed by Hethcote [7] .

The hypotesis $\lambda_{12}=0$ means that the infections on the core people produced by the noncore one is neglected. This is, in our opinion, a reasonable simplification.

Let $D_{1}$ be the domain $x_{1}, y_{1} \geq 0 ; x_{1}+y_{1} \leq 1$ and $D_{2}$ the domain $x_{2}, y_{2} \geq$ $0 ; x_{2}+y_{2} \leq 1$. Then : $D=D_{1} \times \vec{D}_{2}$. By analyzing systems (2) and (3) we obtain the following two threshold parameters :

$$
\sigma_{i}=\frac{\lambda_{i i}\left(\delta_{i}+w_{i}\right)}{\left(\delta_{i}+\gamma\right)\left(v_{i}+\delta_{i}+w_{i}\right)}
$$

$i=1,2$

Lemma 1. a) If $\sigma_{1} \leq 1$, then the only equilibrium point of system(2) in domain $D_{1}$ is the disease-free equilibrium :

$$
\overline{x_{1}}=0 ; \overline{y_{1}}=v_{1} /\left(v_{1}+\delta_{1}+w_{1}\right)
$$

which is globally asymptotically stable in $D_{1}$.

b) If $\sigma_{1}>1$, there are two equilibrium points in $D_{1}$ : the disease free equilibrium $\left(0, \overline{y_{1}}\right)$ and : 


$$
\begin{gathered}
x_{1} *=\frac{\left(\delta_{1}+\gamma\right)\left(v_{1}+\delta_{1}+w_{1}\right)\left(\sigma_{1}-1\right)}{\lambda_{11}\left(\gamma+\delta_{1}+w_{1}\right)} \\
y_{1}=1-\frac{\delta_{1}+\gamma}{\lambda_{11}}\left(1+\frac{\left(v_{1}+\delta_{1}+w_{1}\right)\left(\sigma_{1}-1\right)}{\gamma+\delta_{1}+w_{1}}\right)
\end{gathered}
$$

In this case the point $\left(0, \overline{y_{1}}\right)$ is unstable while point $\left(x_{1} *, y_{1} *\right)$ is globally asymptotically stable in domain $D_{1}-\left\{\left(x_{1}, y_{1}\right): x_{1}=0,0 \leq y_{1} \leq 1\right\}$.

Proof. The equilibrium points are easy to find by analyzing the isoclines

$x_{1}^{\prime}=0, \quad y_{1}^{\prime}=0$.

The Jacobian matrix of $(2)$ at point $\left(0, \overline{y_{1}}\right)$ is :

$$
J\left(0, \overline{y_{1}}\right)=\left(\begin{array}{lr}
\left(\delta_{1}+\gamma\right)\left(\sigma_{1}-1\right) & 0 \\
\left(\gamma-v_{1}\right) & -\left(v_{1}+\delta_{1}+w_{1}\right)
\end{array}\right)
$$

We obtain explicity the eigenvalues of $J\left(0, \overline{y_{1}}\right)$ :

$$
\begin{aligned}
& \lambda_{1}=\left(\delta_{1}+\gamma\right)\left(\sigma_{1}-1\right) \\
& \lambda_{2}=-\left(v_{1}+\delta_{1}+w_{1}\right)
\end{aligned}
$$

We can see that,if $\sigma_{1} \leq 1$, both eigenvalues are negative and if $\sigma_{1} \geq 1$ the eigenvalue $\lambda_{1}$ is positive.In this last case the equilibrium point $\left(0, \overline{y_{1}}\right)$ is a saddle point where the stable manifold in $D_{1}$ is the line $\{(0, y): 0 \leq y \leq 1\}$. If $\sigma_{1}=1$, then the point $\left(0, \overline{y_{1}}\right)$ is the only equilibrium point in the invariant set $D_{1}$ ( is coincident with the point $\left.\left(x_{1} *, y_{1} *\right)\right)$. Applying Dulac's test [10] to the function $\rho(x, y)=1 / x$, we see that there is no periodic orbits in $D_{1}$

The Jacobian matrix of $(2)$ at point $\left(x_{1} *, y_{1} *\right)$ is :

$$
J\left(x_{1 *}, y_{1} *\right)=\left(\begin{array}{cr}
-\gamma_{11} x_{1 *} & -\gamma_{11} x_{1} * \\
\gamma-v_{1} & -\left(v_{1}+\delta_{1}+w_{1}\right)
\end{array}\right)
$$

The eigenvalues are the solution of the characteristic equation :

$$
\lambda^{2}+\lambda\left(\lambda_{11} x_{1} *+v_{1}+\delta_{1}+w_{1}\right)+\lambda_{11} x_{1} *\left(\gamma+\delta_{1}+w_{)}=0\right.
$$

Since $\lambda_{11} x_{1} *\left(\gamma+\delta_{1}+w_{1}\right)>0$, both eigenvalues have negative real parts , hence the point $\left(x_{1} *, y_{1} *\right)$ is locally asymptotically stable. Since there is no periodic orbits , this point is globally stable

In order to analize the non autonomous system (3) we consider the associate autonomous system : 


$$
\begin{gathered}
x_{2}^{\prime}=\left(1-x_{2}-y_{2}\right)\left(\lambda_{22} x_{2}+\lambda_{21} p x_{1}(\infty)\right)-x_{2}\left(\delta_{2}+\gamma\right) \\
y_{2}^{\prime}=v_{2}+\left(\gamma-v_{2}\right) x_{2}-\left(v_{2}+\delta_{2}+w_{2}\right) y_{2}
\end{gathered}
$$

where $x_{1}(\infty)$ is the asymptotic value of $x_{1}(t)$ in system (2) . Note that,by Lemma $1, x_{1}(\infty)=0$ or $x_{1}(\infty)=x_{1} *$

Lemma 2. a) If $x_{1}(\infty)=0$ then two possibilities arise :

i) If $\sigma_{2} \leq 1$, then the only equilibrium point of system (7) in domain $\mathrm{D}_{2}$ is the disease-free equilibrium :

$$
\overline{x_{2}}=0 \quad ; \quad \overline{y_{2}}=v_{2} /\left(v_{2}+\delta_{2}+w_{2}\right)
$$

which is globally asymptotically stable in $D_{2}$.

ii) If $\sigma_{2}>1$, there are two equilibrium points in $D_{2}$ : the disease free equilibrium $\left(0, \overline{y_{2}}\right)$ and :

$$
\begin{gathered}
x_{2} *=\frac{\left(\delta_{2}+\gamma\right)\left(v_{2}+\delta_{2}+w_{2}\right)\left(\sigma_{2}-1\right)}{\lambda_{22}\left(\gamma+\delta_{2}+w_{2}\right)} \\
y_{2 *}=1-\frac{\delta_{2}+\gamma}{\lambda_{22}}\left(1+\frac{\left(v_{2}+\delta_{2}+w_{2}\right)\left(\sigma_{2}-1\right)}{\gamma+\delta_{2}+w_{2}}\right)
\end{gathered}
$$

In this case the point $\left(0, \overline{y_{2}}\right)$ is unstable while point $\left(x_{2} *, y_{2} *\right)$ is globally asymptotically stable in domain $D_{2}-\left\{\left(x_{2}, y_{2}\right): x_{2}=0,0 \leq y_{2} \leq 1\right\}$

b) If $x_{1}(\infty)=x_{1} *$ then there is only one equilibrium point in domain $D_{2}$. This point is the inner point $\left(\hat{x}_{2}, \hat{y_{2}}\right)$ of $D_{2}$, where $\hat{x}_{2}$ is the positive root of the quadratic equation :

$$
\begin{array}{r}
\lambda_{22}\left(\gamma+\delta_{2}+w_{2}\right) x^{2}+ \\
+\left[\left(v_{2}+\delta_{2}+w_{2}\right)\left(\delta_{2}+\gamma\right)-\lambda_{22}\left(\delta_{2}+w_{2}\right)+\lambda_{21} p\left(\gamma+\delta_{2}+w_{2}\right) x_{1} *\right] x \\
-\lambda_{21} p\left(\delta_{2}+w_{2}\right)=0
\end{array}
$$

and

$$
\hat{y_{2}}=\left(v_{2}\left(1-\hat{x_{2}}\right)+\gamma \hat{x_{2}}\right) /\left(v_{2}+\delta_{2}+w_{2}\right)
$$

This point is globally asymptotically stable in $D_{2}$. 
Proof. Note that system(7) with $x_{1}(\infty)=0$ is the same system(2) by substituting suscript 2 in all parameters.

If $x_{1}(\infty)=x_{1}$ * the only suitable equilibrium point is given by the intersection of the isoclines

$$
\begin{aligned}
& x_{2}^{\prime}=0: y_{2}=1-x_{2}-\left(\delta_{2}+\gamma\right) x_{2} /\left(\lambda_{21} p x_{1} *+\lambda_{22} x_{2}\right) \\
& y_{2}^{\prime}=0: y_{2}=-x_{2}+\left(1-\left(\delta_{2}+\gamma\right) / \lambda_{11}\right)
\end{aligned}
$$

By applying Dulac's Lemma to the function $\rho(x, y)=1 / x$ we prove that there are no periodic orbits in $D_{2}$.

We are going to analyze the non autonomous system (3) by using some results of Markus [12] on asymptotically autonomous systems .(See also Thieme [13]) . Let us consider the systems

$$
\begin{array}{r}
x^{\prime}=f(t, x) \\
y^{\prime}=g(y)
\end{array}
$$

in $R^{n}$.System (12) is called asymptotically autonomous, with limit equation (13) , if

$f(t, x) \longrightarrow g(x)$ for $t \longrightarrow \infty$,

locally uniformly in $x \in R^{n}$, i.e., for $x$ in any compact susbset of $R^{n}$.

Theorem 1 [Markus]. Let (12) asymptotically autonomous with limit (13), $f$ continous and locally Lipschitz in $x, g$ continously differentiable, $y *$ a locally asymptotically stable equilibrium of (13). Then there exists $r>0$ and $T_{1}>0$ such that the solution $\phi\left(t, T_{1}, \zeta\right)$ of $(12)$ starting at $\zeta,|\zeta-y *|<r$ at time $T_{1}$ converges to $y *$ for $t \longrightarrow \infty$.

By using this theorem we show the following Lemma :

Lemma 3. Let $H$ be a compact set, positively invariant for systems (12) and (13) ,$y * \in H$ be the globally asymptotically stable equilibrium point in $H$ for system (13). Then $\lim _{t \rightarrow \infty} \phi(t, 0, \zeta)=y *, \forall \zeta \in H$.

Proof. Let $\psi(t, T, \zeta)$ be the solution of (13) starting at $\zeta$ at time $\mathrm{T}$, and let $r>0$ according to Markus Theorem . Due to the compacity of $H$ and the continous dependence on the initial conditions, we can find a time $t_{1}$, not depending on $\zeta$, such that $|\psi(t, 0, \zeta)-y *|<r / 2, \forall t>t_{1}, \forall \zeta \in H$. Let $L>0$ such that $|g(y)-g(x)| \leq L|y-x|$. Than there is a time $t_{2}$ such that

$|g(x)-f(t, x)|<\left(r \exp \left(-t_{1} L\right)\right) / 2 t_{1}, \forall t>t_{2}, \forall x \in H$. Now, for an arbitrary $\zeta \in H$, let $\phi(t)=\phi(t, 0, \zeta), \psi(t)=\psi\left(t, t_{2}, \phi\left(t_{2}\right)\right)$. Then : 


$$
\begin{aligned}
& \left|\phi(t)-\psi^{\prime}(t)\right| \leq \int_{t_{2}}^{t} \mid f(u, \phi(u))-g(\psi(u) \mid d u \leq \\
& \leq \int_{t_{1}}^{t_{1}+t_{2}} \mid f\left(u, \phi(u)-g(\phi(u))\left|d u+L \int_{t_{1}}^{t}\right| \phi(u)-\psi(u) \mid d u \quad \forall t \in\left[t_{1}, t_{1}+t_{2}\right] .\right.
\end{aligned}
$$

lising Gronwall's inequality :

$$
|\phi(t)-\psi(t)| \leq\left[\int_{t_{1}}^{t+t_{2}}|f(u, \phi(u))-g(\phi(u))| d u\right] \exp L\left(t-t_{1}\right) \leq r / 2
$$

Hence, $\left|\phi\left(t_{1}+t_{2}\right)-y *\right|<r$ and the result follows from Markus Theorem Since domain $D_{2}$ is invariant for both systems (3) and (7) and system (3) is asymptotically autonomous with limit (7), then the main stability results given in Lemma 2 for the autonomous system (7) are valid for the nonautonomous system (3). We can sumarize our results in the following Theorem.

Theorem 2. Let $\lambda_{12}=0$ in the four dimensional system (1).Then :

a) If $\sigma_{1} \leq 1$ Then :

If $\sigma_{2} \leq 1$, then the only equilibrium point of (1) in domain $D$ is the disease-free equilibrium: $\left(0, \overline{y_{1}}, 0, \overline{y_{2}}\right)$ which is globally asymptotically stable in $D$.

b) If $\sigma_{1}>1$. Then :

i) If $\sigma_{2} \leq 1$, there are two equilibrium points in domain $D$ : the disease free point $\left(0, \overline{y_{1}}, 0, \overline{y_{2}}\right)$, which is unstable, and the endemic point $\left(x_{1} *, y_{1} *, \hat{x}_{2}, \dot{y}_{2}\right)$ which is globally asymptotically stable in

$D-\left\{\left(0, y_{1}, x_{2}, y_{2}\right): 0 \leq y_{1} \leq 1,\left(x_{2}, y_{2}\right) \in D_{2}\right\}$

ii) If $\sigma_{2}>1$, there are three equilibrium points: the disease free point $\left(0, \overline{y_{1}}, 0, \overline{y_{2}}\right)$ which is unstable, the point $\left(0, \overline{y_{1}}, x_{2}, y_{2} *\right)$ which is also unstable, and endemic point $\left(x_{1} *, y_{1} *, \hat{x_{2}}, \hat{y_{2}}\right)$ which is globally asymptotically stable in

$$
D-\left\{\left(0, y_{1}, x_{2}, y_{2}\right): 0 \leq y_{1} \leq 1,\left(x_{2}, y_{2}\right) \in D_{2}\right\}
$$

\section{REMARKS.-}

a) The condition $\sigma_{1}>1$ implies that the core population can maintain an endemic level in the whole population, even when the non-core satisfies $\sigma_{2} \leq 1$. In this case the endemicity on the non-core is maintained only by the core.

b) The case : $\sigma_{1} \leq 1, \sigma_{2}>1$ has been excluded of the Theorem because Lemma 3 is not applicable (the invariant compact set $D_{2}$ is not the domain of attraction of the non trivial equilibrium point due to the existence of a second equilibrium point on the boundary). In this case there are two equilibrium points: $\left(0, \overline{y_{1}}, 0, \overline{y_{2}}\right)$ and $\left(0, \overline{y_{1}}, x_{2} *, y_{2} *\right)$. The first is unstable while the second one seems to be globally asymptotic ally stable in domain: $D-\left\{\left(0, y_{1}, x_{2}, y_{2}\right): 0 \leq y_{1} \leq 1,\left(x_{2}, y_{2}\right) \in D_{2}\right\}$

In this case the endemicity on the non-core population is maintained by itself, without the action of the core. 
c) The point $\left(x_{1} *, y_{1} * 0,0\right)$ is not an equilibrium point of the system in all the cases. That means only that the core is not isolated, so that any endemicity of the core is transfered to the non-core.

d) Parameter estimation and some illustrative computer simulations can be founded in [3].

Acknowledgements. I thank H. Hethcote for several critical remarks

\section{REFERENCES}

[1] E.Beretta ; V. Cappaso : Global stability results for a multigroup SIR Epidemic Model. Proc of the Autumn Course Reseach Seminars on Mathematical Ecology . ICTP, Trieste 1986.

[2] González-Guzmán,Jorge:An epidemiological model for direct and indirect transmission of typhoid fever. Math.Biosc.96,33:-46,1989.

[3] Crollzález-Guzmản,Jorge ;González -Yánez ,Betsabé : Local Stability Results on a Model for Typhoid Fever with a Core Group. Proyecciones, Vol 12 ;N 2 ;161-169;(1993)

[4] González-Guzmán,Jorge ;Naulin,Raúl : Analysis of a model of bovine brucellosis using singular perturbations. (Accepted in Journal of Math. Biol. 1994)

[5] H.Hethcote ;J.Yorke: Gonorrhea:Transmission Dynamics and Control. Lecture Notes in Biomathematics 56.Springer Verlag 1984

[6] H.Hethcote :Qualitative analysis of communicable disease models. Math.Biosc.28,335-356,1976.

[7] H.Hethcote :An immunization Model for a Heterogeneous Population. Theoret.Population Biol. .14:338-349 (1978)

[8] H.Hethcote; H.Thieme : Stability of the Endemic Equilibrium in Epidemic Models with Subpopulations. Math.Biosc.75:205-227(1985)

[9] H.Hethcote ;J.van Ark : Epidemiological Models for Heterogeneous Populations: Proportionate Mixing,Parameter Estimation, and Immunization Programs. Math.Biosc.84:85-118(1987)

[10] D.W.Jordan and Smith, Nonlinear Ordinary Differential Equations,Oxford Univ.Press, 1977 .

[11] M.Levine; Catterine Ferreccio;R.Black;Carol Tacket;R. Germanier. Progress in Vaccines Against Typhoid Fever. R.of Infectious Diseases, Vol.11 May-June 1989 . 
[12] Markus,L. Asymptotically autonomous differential systems. In: Lefschetz,S(ed.).Contribution to the Theory of Nonlinear Oscillations III Princeton Univ.Press 1956.

[1.3] Thieme,Horst : Convergence results and a Poincare-Bendixon trichotomy for asymptotically autonomous differential equations. J.Math.Biol (1992)30:755763.

[14] H.R.Thieme,Horst : Global asymptotic stability in epidemic models . Equadiff , Lecture Notes in Math. 1017, Springer Verlag ,Heildelberg ,1983 , pp. 608615 .

Received: March 24, 1994

Jorge González-Guzmán

Instituto de Matemática

Universidad Católica de Valparaíso

Casilla 4059, Valparaiso - Chile. 